\title{
BMJ Open Distribution and extent of electronic medical record utilisation in eye units across the United Kingdom: a cross- sectional study of the current landscape
}

\author{
Shin Bin Lim, ${ }^{1}$ Humma Shahid ${ }^{2}$
}

To cite: Lim SB, Shahid H. Distribution and extent of electronic medical record utilisation in eye units across the United Kingdom: a cross-sectional study of the current landscape. BMJ Open 2017;7:e012682. doi:10.1136/ bmjopen-2016-012682

- Prepublication history and additional material are available. To view these files please visit the journal online (http://dx.doi.org/ 10.1136/ bmjopen-2016-012682).

Received 17 May 2016 Revised 13 March 2017 Accepted 14 March 2017

CrossMark

${ }^{1}$ University of Cambridge,

Cambridge, UK

${ }^{2}$ Department of Ophthalmology, Addenbrooke's Hospital,

Cambridge, UK

Correspondence to Dr. Humma Shahid;

humma.shahid@addenbrookes. nhs.uk

\section{ABSTRACT}

Objectives Ophthalmology units across the UK vary widely in their adoption of electronic medical records (EMR). There is a lack of evidence to show the extent and progress of EMR adoption. The aim of this study was to capture a snapshot of the current landscape of EMR use, as a baseline for comparison in future studies.

Setting An electronic survey questionnaire was sent to all NHS ophthalmology Units in the UK.

Participants A total of 104 National Health Service (NHS) ophthalmology units participated in the survey, which was carried out over 6 months from December 2013 to June 2014.

\section{Primary and secondary outcome}

measures Respondents were asked about technology usage pertaining to specific processes in the clinic workflow. This allowed us to determine the extent of EMR usage and details about current use or planned implementation by each unit.

Results $77.6 \%(n=104)$ of NHS ophthalmology units responded. $45.3 \%$ ( $n=48)$ of units were currently using an EMR and a further $26.4 \%(n=28)$ of units plan to implement EMR within 2 years. $70.8 \%$ of units with a current EMR system use Medisoft. EMR is used by all clinicians in $37.5 \%$ and by all subspecialties offered at the unit in $27.0 \%$. In $56.3 \%$, new clinical notes are entered into EMR only by clinicians. All imaging devices are networked to EMR in $28.3 \%$. In $46.7 \%$, EMR is accessible by other specialties within the same hospital. $71.1 \%$ would recommend EMR to a colleague.

Conclusions EMR has the potential to address current limitations of patient information transfer and sharing in ophthalmology. It is pleasing to see a significant proportion of units already engaging with EMR or having plans to do so in the near future. However, differing EMR systems and lack of remote access mean further optimisation of these record systems are needed to allow data transfer between units.

\section{INTRODUCTION}

The past decade has seen electronic medical records (EMR) slowly permeate medical practice in the UK. There is a significant financial incentive for the NHS to embrace this revolution and help it to deliver health services sustainably in a time of real financial pressure ${ }^{1}$
Strengths and limitations of this study

First comprehensive study of use of electronic medical records (EMR) in ophthalmology in the UK with high participation rate $(78 \%)$ and good distribution of responding units.

- Quantitative and qualitative data obtained from questionnaire targeted at specific points of clinic workflow.

- Responses predominately obtained via the internet, which may preselect for more technologically advanced practices.

- Responses filled up by a single clinician in the department whose view may not be representative of the whole department.

with the use of EMR, electronic prescribing and referrals by electronic mail. An EMR also has the potential to meet the evolving needs of changing population demographics ${ }^{2}$ where the number of patients with chronic medical conditions has grown dramatically, across multiple locations who each need information at the point of care. EMRs can facilitate the rapid storage, transmission and retrieval of patient data, thereby improving delivery of integrated care to patients. In addition, EMR is crucial for the collection of data for research and audits, which underpin modern medical practice. An example is the National Ophthalmology Database (NOD) Audit managed by The Royal College of Ophthalmologists collated pseudoanonymised data from EMRs and extracted details of over 480000 clinical episodes including cataract surgery, hospital diabetic care, age related macular degeneration (AMD) treatments and glaucoma surgery. ${ }^{3}$ This valuable ability to combine vast amounts of data across sites has not been demonstrated by any other EMR to date and allows for appraisal, revalidation, audit and research. 
Ophthalmology is a specialty that involves the constellation of medical and surgical care, heavy reliance on interpretation of diagnostic imaging, high-volume clinical practices with complex workflows and documentation requirements involving a combination of numerical, textbased and image-based data elements. ${ }^{4}$ All these factors make ophthalmology well suited to the use of EMR. EMR also allows access of a patient's ophthalmological data by other specialties at the point of care.

The next few years will see much progress made in the use of EMR in ophthalmology. It is unlikely that a single system will be adopted universally as previous attempts to do this have led to technical difficulties and lack of success. ${ }^{5-8}$ This study intends to capture a snapshot of the landscape at this moment in time by studying the distribution of EMR use and the extent to which it is used in various eye units. It is hoped that these results can be used as a baseline for comparison of EMR adoption in 10 or 20 years' time and that our findings may be used as a springboard for more in-depth study into EMR use in ophthalmology in the UK. There is also significant interest in ophthalmology EMR usage overseas, and it is hoped that this study will be used for comparison and sharing of lessons from EMR implementation between countries.

\section{MATERIALS AND METHODS}

This study followed the tenets of the Declaration of Helsinki. This study did not involve the participation of patients or the use of patient information hence ethics approval was not required. Informed consent from all clinicians answering the survey questionnaire was obtained.

\section{Survey instrument}

To assess the adoption of EMR systems in ophthalmology, a literature search and clinical experience were used to devise an appropriate questionnaire. The scientific databases PubMed, Cochrane and MedlinePlus were searched for using the terms 'electronic medical records', 'electronic health records', 'ophthalmology' and 'information technology' for previous analyses of EMR both within ophthalmology and in other specialties. ${ }^{9-14}$ The survey was designed and administered by the web-based programme SurveyMonkey. The complete survey was accessible online at http://www.surveymonkey.com/ s/EMROph for the period of the survey. Questions are listed in table 1.

\section{Survey population}

To provide a geographically representative population of ophthalmologists, a list of all the eye units in the UK was obtained from The Royal College of Ophthalmology website. Each eye unit was contacted for a response. Where more than one response was submitted from the same unit, the additional responses were not included for analysis.

\section{Survey administration}

The college tutor of each eye unit was contacted by email and asked to fill the web-based survey via a link in the email. Follow-up messages were sent to those units who had not responded within 1 month and again at 2 months. For those who had not responded within 2 months, an alternative clinician in the unit was contacted and reminded after 1 month. For those units that still had not responded after that, the clinicians were offered the opportunity to complete the survey as free text within the email rather than having to go to an external link or have a hard copy of the survey posted to them. This was to allow units that may not be so technologically advanced an opportunity to participate in the survey as well. These efforts persisted until over $75 \%$ of the sample population had responded. The total period of the survey lasted from December 2013 to June 2014. The results were collected and compiled electronically using the SurveyMonkey software.

\section{RESULTS}

Of the 134 units approached to participate in the survey, responses were received from $104(78 \%)$. The full list of responding units is placed in the online supplementary appendix. Figure 1 show the geographical distribution of respondents and extent of usage.

A percentage of $45.3(n=48)$ of respondents reported that they were currently using an EMR system in their eye unit. A further $26.4 \% \quad(\mathrm{n}=28)$ reported that they did not currently have an EMR system but planned to implement this within the next 2 years. The remaining $28.3 \%(n=30)$ did not currently have an EMR system and had no plans to implement this within the next 2 years.

\section{EMR system used or planned for implementation}

Respondents were asked to state the name of the EMR software they are using or planning to implement. The results are shown in figure 2.

Medisoft is the most commonly used software. Currently, it is used by $70.8 \%$ ( $n=34$ ) of units with an EMR system. A further six units are planning to implement Medisoft within the next 2 years. If implementation in all units goes to plan, the percentage of Medisoft users in 2 years will fall to $52.6 \%(\mathrm{n}=40)$, but it will still remain the most widely used software. OpenEyes is currently used by $8.3 \%$ $(n=4)$ of units, but in 2 years it will rise to $18.4 \%(n=10)$, making it the second most commonly used software. One unit reported currently using a mixture of Medisoft and a trust-wide software and another reported using mixture of OpenEyes and a trust-wide software.

\section{EMR use in existing units}

The 48 units that had already an EMR in place were asked further questions on how the system was used within their unit. A percentage of 37.5 of units reported that EMR was used by all clinicians, while $62.5 \%$ reported that EMR was used only by some clinicians. With regard to usage by subspecialty, $27.0 \%$ reported that EMR was used by all 


\begin{tabular}{l} 
Table 1 Survey questionnaire \\
\hline $1 \quad \begin{array}{l}\text { Question } \\
\text { department: }\end{array}$ \\
\hline $2 \quad \begin{array}{l}\text { The name of our EMR system currently used or the one } \\
\text { planned for implementation is: }\end{array}$
\end{tabular}

3 The EMR system is used by:

4 The EMR system is used by:

$9 \quad$ Imaging devices at your unit (eg,

OCT, visual fields) are:

$6 \quad$ Historical data from paper records is

7 New clinical documentation is entered by:

8 Clinical documentation on the EMR includes the following:
5 The EMR system is used by:

\section{Options}

- We currently use an EMR system

- We do not currently have an EMR system but plan to implement this in the next 2 years

- We do not currently use an EMR system

If you have selected the last option 'we do not currently use an EMR system', you may submit the survey now

(Please state)

If you have selected 'we do not currently have an EMR system but plan to implement this in the next 2 years', you may submit the survey now

- All clinicians

- Some clinicians

- All subspecialties available at unit

- Some subspecialties available at unit

Yes/No/NA if subspecialty not offered at the unit

- Primary care

- Glaucoma

- Cornea

- Medical retina

- Surgical retina

- Oculoplastics

- Paediatrics

- Medical ophthalmology

- Neuro-ophthalmology

- Manually entered by clinicians ad hoc

- Available on request

- Not available

- Clinicians

- Non-clinicians

- Presenting complaint

- Medications

- Allergies

- Smoking status

- Drug allergy alerts

- Drug interaction alerts

- Management plan

- All networked (state name of networking system)

- Some are networked (state name of networking system)

- Not networked but there are plans for networking

- Not networked; no plans for networking

10 Transfer of images from imaging devices to the EMR is done by:

- Direct sharing electronically

- Manual scanning of hard copy image into EMR

- Manual transcribing of data into EMR

- Mix of direct electronic sharing and manual methods

11 EMR of your unit is also accessible by:

- Other specialties within same hospital

- GPs in region

- Other units (ophthalmology and non-ophthalmology) across the country

- Public health organisations

- External vendor

- Hospital IT department

- Other (please specify) 


\begin{tabular}{|c|c|c|}
\hline & Question & Options \\
\hline 13 & Devices used for EMR are: & $\begin{array}{l}\text { Desktop/laptop at fixed place } \\
\text { Mobile laptops brought around various work areas } \\
\text { Tablet (including iPad) } \\
\text { Smartphone } \\
\text { Other (please specify) }\end{array}$ \\
\hline 15 & I feel that: & $\begin{array}{l}\text { Agree/disagree/no difference } \\
\text { EMR saves time } \\
\text { EMR improves patient care } \\
\text { Clinical records are captured accurately by EMR } \\
\text { I would recommend EMR to a colleague }\end{array}$ \\
\hline 16 & $\begin{array}{l}\text { Have any in-house studies been done on effect of EMR } \\
\text { on productivity? }\end{array}$ & $\begin{array}{l}>\text { Yes } \\
>\text { No }\end{array}$ \\
\hline 17 & $\begin{array}{l}\text { Are you happy for us to contact you regarding the } \\
\text { results of your study (if applicable)? }\end{array}$ & $\begin{array}{l}>\text { Yes } \\
>\text { No }\end{array}$ \\
\hline
\end{tabular}

EMR; electronic medical records; GPs, general practitioners; OCT, optical coherence tomography.

subspecialties offered at the unit, while $73.0 \%$ reported that EMR was used only by some subspecialties offered at the unit. Figure 3 shows usage by subspecialty. EMR was most extensively used for medical retina and glaucoma and least extensively used for paediatrics and neuro-ophthalmology.

In terms of clinical documentation, new clinical notes are entered into the EMR by clinicians in $56.3 \%$ of the units. In $10.4 \%$, only non-clinicians use the EMR. In $33.3 \%$, both clinicians and allied health professionals

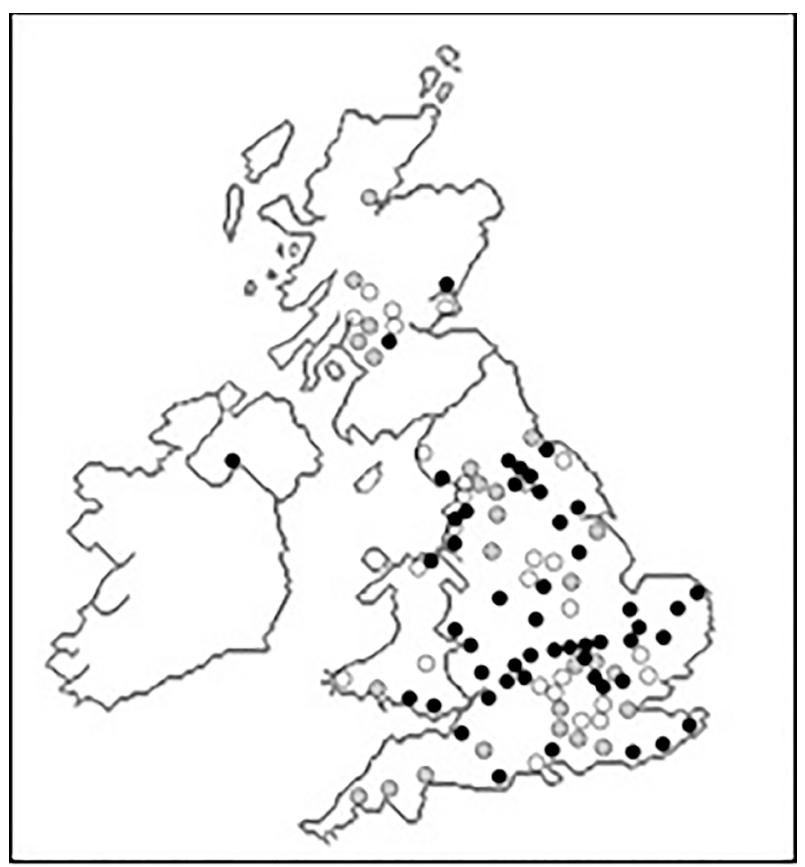

Figure 1 Distribution of respondents and usage of EMR. EMR, electronic medical records. use the EMR. Regarding the content of clinical notes, presenting complaint was documented in $95.6 \%$ of units, medications in $97.8 \%$, allergies in $91.1 \%$, smoking status in $71.1 \%$, drug allergy alerts in $88.9 \%$, drug interaction alerts in $60.0 \%$ and management plan in $93.3 \%$. Historical data from paper records was manually entered by the clinician ad hoc in $62.0 \%$ of units. Historical data were only available on request in $42.0 \%$ and not available at all in $10.0 \%$.

\section{Imaging}

Respondents were asked if imaging devices are networked to the EMR in their units. These results are shown in figure 4.

A range of image networking systems are used. Medisoft is used by $14.6 \%$ of the units, Topcon by $4.1 \%$, local area network by $6.3 \%$, Imagenet by $6.3 \%$, Heidelberg by $6.3 \%$, Optomize by $6.3 \%$ and another system by $14.6 \%$. A percentage of 41.5 units are unsure of what networking systems are used. Images are directly shared electronically in $45.5 \%$ of units. Hard copy images are manually scanned into EMR in $9.1 \%$. Data are manually transcribed into EMR in $2.3 \%$. A mix of direct electronic sharing and manual methods are used in $22.7 \%$ of units. Images are not transferred in $20.4 \%$.

\section{Access to EMR}

One of the advantages of EMR is the ability to store data geographically and between providers. EMR is accessible by other specialties within the same hospital in $46.7 \%$ of units, by general practitioners in the region in $4.4 \%$, by other ophthalmology and non-ophthalmology units across the country in $6.7 \%$ and by public health organisations in $2.2 \%$. 


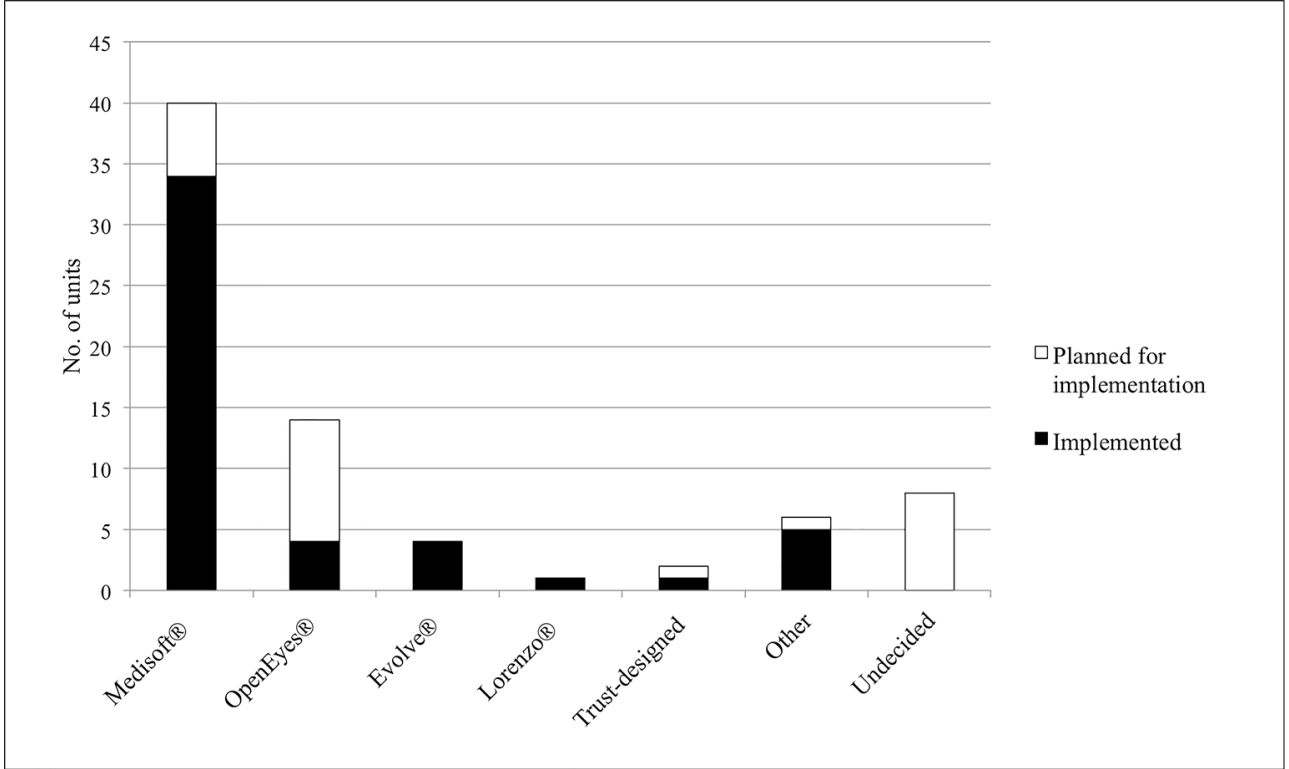

Figure 2 EMR system used or planned for implementation. EMR, electronic medical records.

\section{Devices used for EMR}

The main device used for EMR is a desktop or laptop at a fixed place, used in 100\% of units. Additionally, 29.2\% also use mobile laptops and $10.4 \%$ of units also use tablets. None of the units have EMR on a smartphone portal.

\section{Technical support}

Technical support is only available during working hours on weekdays during the hours of 9:00-17:00 in most cases $(68.9 \%)$. Only $4.4 \%$ of units have 24 -hour technical support, while the remaining $22.2 \%$ were unsure of the hours of their technical support.

\section{Design of EMR}

Nearly all the units $(87.0 \%)$ are using an EMR system designed solely by an external vendor. A percentage of 10.9 are using an EMR system designed by both an external vendor and the hospital's IT department, while $2.2 \%$ of units were using a system designed in-house by the hospital IT department.

\section{Satisfaction with EMR}

Units that had implemented EMR were also asked to rate their satisfaction of the EMR based on four criteria. Overall, most units thought that (1) EMR improves patient care, (2) clinical records are captured accurately by EMR and (3) would recommend EMR to a colleague. However, the majority of units did not think that EMR saves time. The results are shown in figure 5 .

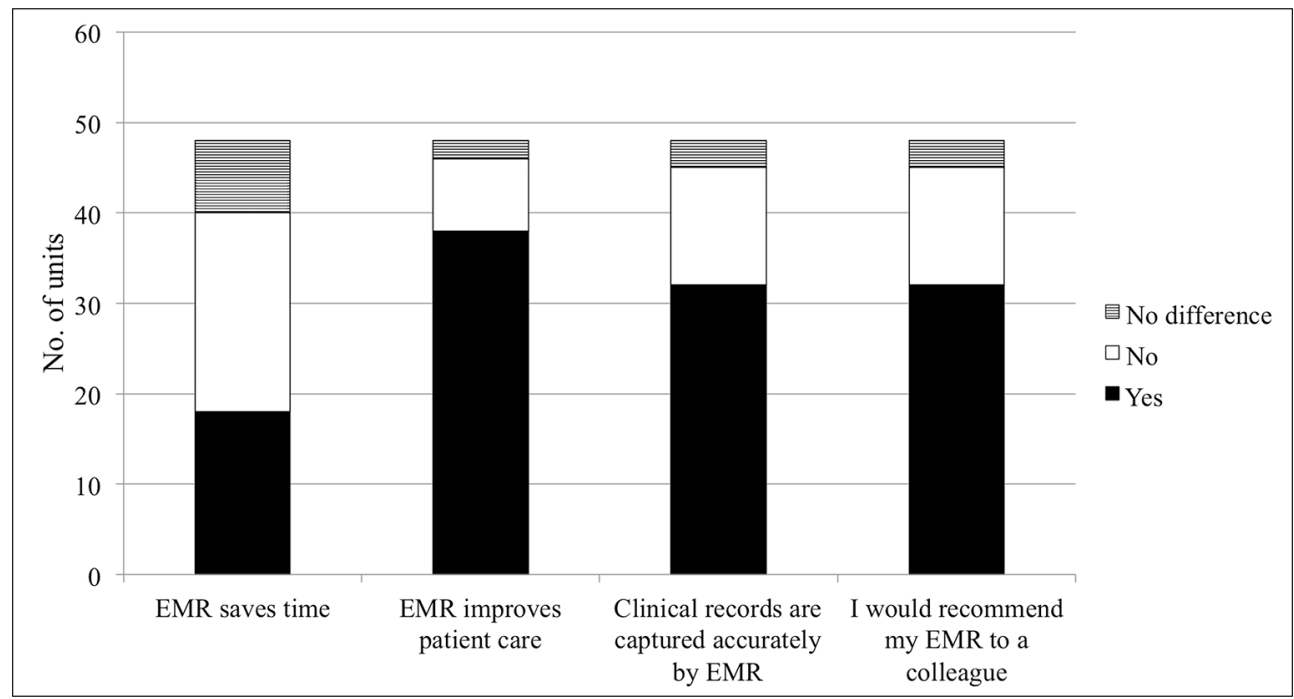

Figure 3 EMR usage by subspecialty. 'Not applicable' indicates that the subspecialty was not offered at the unit. EMR, electronic medical records. 


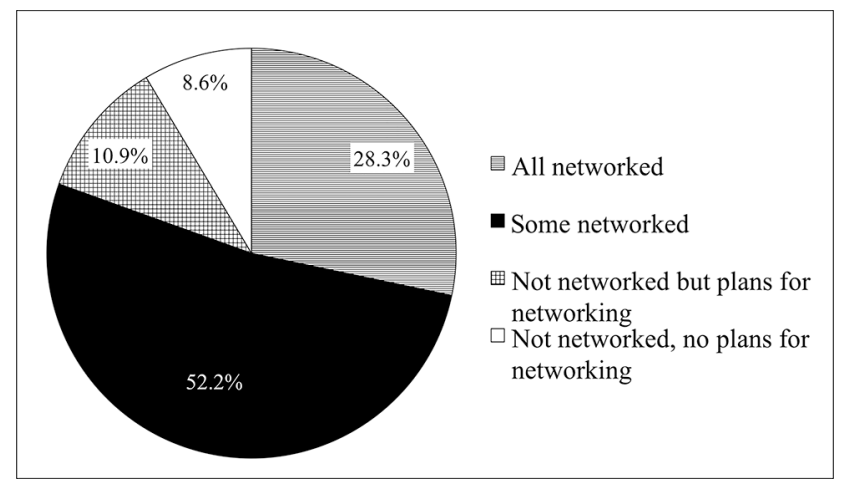

Figure 4 Networking of imaging devices.

\section{DISCUSSION}

This is the first comprehensive study on the use of EMR in ophthalmology units cross the UK. The participation rate was $78 \%$, and the responding units are fairly well-distributed throughout the country. Currently, $45 \%$ of all eye units in the UK are using an EMR system, with its use more widespread in London, East of England, Oxford and West Midlands compared with other parts of the UK. However, taking into account declared plans for EMR implementation, the next 2 years will see a rapid growth in EMR usage to $71.7 \%$ of the eye units surveyed, with areas in the South of England and Scotland seeing a greater uptake of EMR within this timeframe. This reflects significant engagement with government proposals for a paperless NHS by 2018. It is noted that since our survey was performed, The Royal College of Ophthalmologists has selected Medisoft as the EMR to be used throughout England and Wales as part of the National Cataract Audit. ${ }^{3}$ This may mean implementation of this system within all NHS Trusts in England and Wales that do not currently have an EMR, bringing the total Medisoft users to 104 out of 134 units surveyed $(77.6 \%)$ once the uptake of EMR is $100 \%$ in all responding units.

The EMR is used by both doctors and allied health professionals in only $33.3 \%$ of the units. Greater data capture would be possible if the system were used universally by all staff. There is also a deficiency in image networking within ophthalmology departments. Only $28.3 \%$ units have all images networked, and less than half of the units $(45.5 \%)$ are currently able to share images directly from devices to the EMR by electronic upload. Given the importance of ophthalmic imaging and automated perimetry, it is essential for any EMR system to be integrated with an image networking system.

Where EMR exists, it is not universally adopted by all clinicians within that unit. EMR was used by some but not all clinicians in $62.5 \%$ of units, and used in some but not all subspecialties $73.0 \%$ of units. This may reflect unfamiliarity with the EMR system or preference for documenting notes in paper format, particularly as EMR has a negative impact on patient throughput in a high-volume outpatient specialty. Many ophthalmologists feel that the intricacies of a thorough ophthalmic examination and sometimes detailed drawings cannot be adequately or quickly recorded with a series of dropdown menus and prefabricated electronic picture tools that are typically more time consuming and less accurate than freehand drawings. ${ }^{15}$ In our study, only $37.5 \%$ of units stated that EMR saves time, but $67 \%$ of units stated that EMR documents clinical notes accurately. Clinicians appear to perceive that accuracy of documentation is less of an issue than the time spent on EMR. This is something that ophthalmology EMR systems will need to improve in order to increase productivity and uptake.

Remote access to EMR data is not universal, with $35.4 \%$ of units not allowing access of EMR records outside of the hospital environment. This is in part due to security advice from hospital information technology departments to protect patient data. With the government's push towards transparency of information in this digital age and allowing public to access health records online, ${ }^{16}$ a fine balance will have to be struck between greater sharing of information and more robust methods of data protection to ensure that patient confidentiality is not breached.

Overall, it would appear that most clinicians are largely satisfied with EMR. The majority reported that (1) EMR

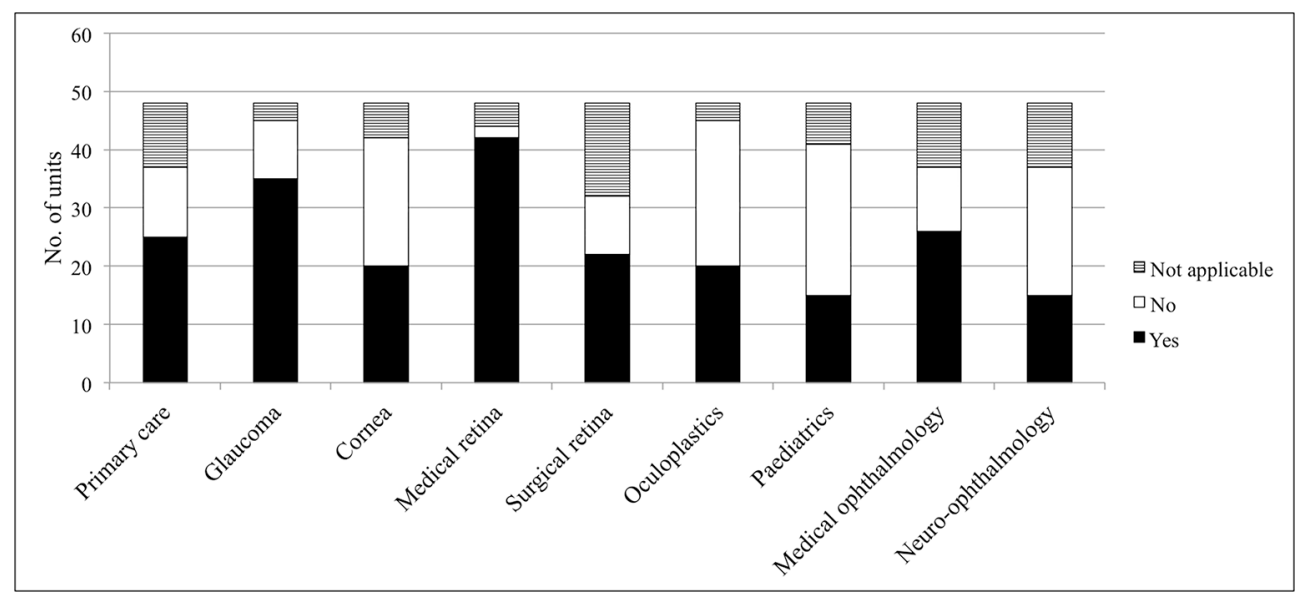

Figure 5 Respondents' satisfaction with EMR. EMR, electronic medical records. 
improves patient care, (2) clinical records are captured accurately by EMR and (3) would recommend EMR to a colleague. However, majority of units did not think that EMR saves time. It would be useful to study the productivity of EMR to determine if EMR improves patient outcomes, and whether different platforms are comparable.

A limitation of our study is the questionnaire design that did not include scoring for questions to rate satisfaction of the local EMR system. We limited the survey to ophthalmologists, but the quality of the data could be enhanced through interviews with IT staff. Nevertheless, the response rate was good, and it was felt that the data retrieved is of valuable quality.

There are more than six EMR systems in use, of which Medisoft and OpenEyes are the most widely used. Both OpenEyes and Medisoft are EMR systems solely for ophthalmology. The variation in software systems used may pose some difficulties in terms of sharing information between eye units. Our study has found that in terms of content, most EMR platforms allow documentation of presenting complaint, medications, allergies and management plan, and it is noted that nearly all of these systems comply with the dataset guidelines issued by The Royal College of Ophthalmologists for the purpose of the NOD Audit. However, a future study would be required to examine in greater detail how these different EMR platforms differ in outcomes and productivity. From our study, it appears that EMR is primarily used at fixed desktop or laptop devices and less so on mobile or tablet devices. It is likely that a future study will notice a significant trend towards mobile data entry and access when compared with the data we have captured.

\section{CONCLUSION}

In conclusion, EMR use in ophthalmology is set to increase further in the coming years. This snapshot of the landscape of EMR usage across the UK has shown that a significant proportion of units are already engaging with EMR or have plans to do so in the near future. These initial findings pave the way for more studies on productivity assessment and comparison across units and can be used as a baseline with which to compare EMR use a few years down the line. Our study has also found that the extent and way of usage varies greatly across units. Perhaps there is now room for sharing of experience and ideas between units, which could allow units to reap greater economies of scale, increase productivity and ultimately deliver the benefits to our patients.

Acknowledgements The authors would like to thank all responding units for their participation in our survey.
Contributors SBL drafted the survey questionnaire, analysed the results and drafted the manuscript. HS edited the survey questionnaire and final manuscript. Both SBL and HS contacted the eye units for responses.

Competing interests None declared.

Provenance and peer review Not commissioned; externally peer reviewed.

Data sharing statement Raw data on survey responses can be obtained by contacting the authors at the corresponding address.

Open Access This is an Open Access article distributed in accordance with the Creative Commons Attribution Non Commercial (CC BY-NC 4.0) license, which permits others to distribute, remix, adapt, build upon this work non-commercially, and license their derivative works on different terms, provided the original work is properly cited and the use is non-commercial. See: http://creativecommons.org/ licenses/by-nc/4.0/

(c) Article author(s) (or their employer(s) unless otherwise stated in the text of the article) 2017. All rights reserved. No commercial use is permitted unless otherwise expressly granted.

\section{REFERENCES}

1. PricewaterhouseCoopers LLP. A review of the potential benefits from the better use of information and technology in Health and Social Care: final report. London, 2013.

2. Chiang MF, Boland MV, Margolis JW, et al. Adoption and perceptions of electronic health record systems by ophthalmologists: an American Academy of Ophthalmology survey. Ophthalmology 2008;115:1591-7.

3. Day AC, Donachie PH, Sparrow JM, et al. The Royal College of Ophthalmologists' National Ophthalmology Database study of cataract surgery: report 1, visual outcomes and complications. Eye 2015;29:552-60.

4. Chiang MF, Boland MV, Brewer A, et al. Special requirements for electronic health record systems in ophthalmology. Ophthalmology 2011;118:1681-7.

5. Department of Health. The National Programme for IT in the NHS: an update on the delivery of detailed care records systems, 2011.

6. National Audit Office (NAO). Department of Health: the National Programme for IT in the NHS. HC 1173 [report] London. UK: The Stationery Office Ltd, 2005.

7. Parliament UK. Evidence submitted by Alan Shackman (EPR 38). http://www.publications.parliament.uk/pa/cm200607/cmselect/ cmhealth/422/422we67.htm\#n108 (accessed 17 May 2016).

8. The National Programme for ITin the NHS-A Case History. Cambridge, England: University of Cambridge, 2014. http://www. cl.cam.ac.uk/ rja14/Papers/npfit-mpp-2014-case-history.pdf (accessed 17 May 2016).

9. Sanders DS, Lattin DJ, Read-Brown S, et al. Electronic health record systems in ophthalmology: impact on clinical documentation. Ophthalmology 2013;120:1745-55.

10. Boland MV, Chiang MF, Lim MC, et al. Adoption of electronic health records and preparations for demonstrating meaningful use: an American Academy of Ophthalmology survey. Ophthalmology 2013;120:1702-10.

11. Chan P, Thyparampil PJ, Chiang MF. Accuracy and speed of electronic health record versus paper-based ophthalmic documentation strategies. Am J Ophthalmol 2013;156:165-72.

12. Kokkonen EW, Davis SA, Lin HC, et al. Use of electronic medical records differs by specialty and office settings. J Am Med Inform Assoc 2013;20:e33-e38.

13. Wakamiya S, Yamauchi K. Evaluation of electronic medical records for ophthalmology based on changes in staff work through introduction of electronization. Stud Health Technol Inform 2013;192:968.

14. Pandit RR, Boland MV. The impact of an electronic health record transition on a Glaucoma subspecialty practice. Ophthalmology 2013;120:753-60.

15. Nissman SA. Electronic health records. Ophthalmology 2009;116:1018-9.

16. GOV.UK. https://www.gov.uk/government/news/jeremy-huntchallenges-nhs-to-go-paperless-by-2018-2 (accessed 17 May 2016). 